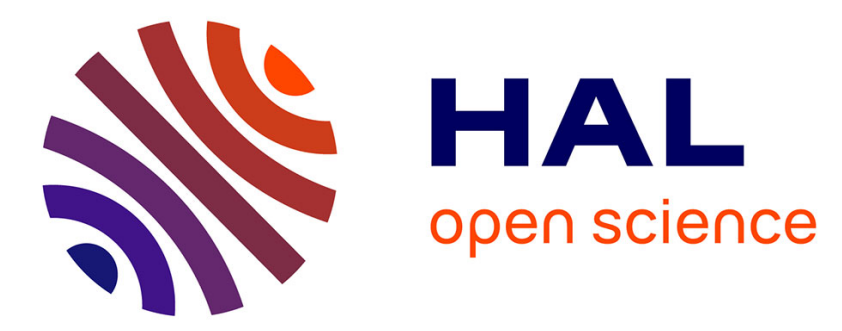

\title{
Les tableaux de bord sur données massives, pour un nouveau management de l'innovation?
}

\author{
Jean-Sebastien Vayre
}

\section{To cite this version:}

Jean-Sebastien Vayre. Les tableaux de bord sur données massives, pour un nouveau management de l'innovation?. Innovations - Revue d'économie et de management de l'innovation, 2015, Capital intellectuel et management de l'innovation, 2 (47), pp.101-121. hal-01227402

\section{HAL Id: hal-01227402 \\ https://hal.science/hal-01227402}

Submitted on 4 Jan 2016

HAL is a multi-disciplinary open access archive for the deposit and dissemination of scientific research documents, whether they are published or not. The documents may come from teaching and research institutions in France or abroad, or from public or private research centers.
L'archive ouverte pluridisciplinaire HAL, est destinée au dépôt et à la diffusion de documents scientifiques de niveau recherche, publiés ou non, émanant des établissements d'enseignement et de recherche français ou étrangers, des laboratoires publics ou privés. 


\title{
LES TABLEAUX DE BORD SUR DONNÉES MASSIVES, POUR UN NOUVEAU MANAGEMENT DE L'INNOVATION?
}

\author{
Jean-Sébastien Vayre (CERTOP - UMR 5044)
}

\section{INTRODUCTION}

Avec le développement des Technologies de l'Information et de la Communication (TIC) dans la sphère du marché (Rallet, 2001) et dans celle de l'organisation (Riccio et Bonnet, 2011), les consommateurs et les travailleurs sont conduits à produire quotidiennement de nombreuses traces numériques renseignant leurs activités de consommation et de fabrication (Kessous, 2012). Ces traces sont souvent dîtes d'usage (Mille, 2013) et constituent autant de données que les managers de l'entreprise nomment les big data ${ }^{1}$.

Dans le contexte de capitalisme cognitif (Boutang, 2007) et de post-taylorisme (Conink, 2004) que nous connaissons actuellement, ces traces d'usage sont intéressantes pour l'entreprise car elles constituent potentiellement une source de valeur ajoutée. En effet, à travers le système de captation (Cochoy, 2004) que composent les technologies big data, les professionnels du marché et de l'organisation peuvent aujourd'hui reconstruire les comportements des consommateurs et des travailleurs de façon à mieux comprendre leur motivation et à mieux prévoir leur action (Vayre, 2013a ; 2014). Autrement dit, en permettant aux managers de l'entreprise de développer des connaissances fines sur les usages que les travailleurs font des systèmes de production et que les consommateurs font des produits/services de consommation, les big data composent un ensemble de matériaux particulièrement utile à la gestion des processus d'innovation (Mallard, 2011).

\footnotetext{
${ }^{1}$ Notons toutefois que les big data ne se réduisent pas aux seules données d'usage. Car, du point de vue des professionnels de l'organisation et du marché, la notion de big data est souvent définie à travers les « $3 \mathrm{~V}$ » (Vayre, 2013a ; 2014). Le premier correspond au volume est désigne la gigantesque quantité de données produites quotidiennement par les entreprises et les personnes. Le second renvoie à la vitesse est décrit la grande rapidité à laquelle ces données sont générées, capturées et partagées. Le troisième est celui de la variété est désigne la multiplicité des sources de production de ces données. Plus précisément et concernant ce dernier point, les big data recouvrent au moins deux grands types de données. Les données d'usage qui sont produites à travers les utilisations que les consommateurs et les travailleurs font des TIC en général (e.g. : les réseaux sociaux et plus largement l'ensemble des sites Internet, l'ensemble des applications Intranet des entreprises, les téléphones portables, les tablettes numériques, les ordinateurs fixes et portables, les objets connectés, etc.). Et, les données scientifiques, techniques et/ou publiques (dont font partie les open data) qui sont produites par différents organismes scientifiques, techniques et/ou publiques (e.g. : Météo-France, l'Insee, les collectivités territoriales, etc.).
} 
Cependant, les traces d'usage doivent d'abord faire l'objet d'une traduction consistant à les « re-présenter $»^{2}$ (Latour, 1993) afin de constituer un réel intérêt pour l'entreprise (Akrich et al., 2006). Car, ce n'est qu'une fois rendues lisibles et comparables que les données d'usage peuvent constituer un avantage concurrentiel. La visualisation des traces d'usage peut en effet permettre aux managers d'adapter plus efficacement, rigoureusement et rapidement leurs innovations organisationnelles et/ou marchandes aux fluctuations des demandes internes et/ou externes à l'entreprise. Et, ce que nous appelons les Tableaux de Bord sur Données Massives (TBDM) ont précisément pour fonction de « re-présenter » (Latour, 1993) les traces d'usage de manière à faciliter leur compréhension et leur manipulation. En d'autres termes, les TBDM sont des technologies de visualisation des données; c'est-à-dire, «des techniques de représentation graphique et d'exploration visuelle de données quantitatives permettant de traduire un ensemble de données brutes en information [...] pour améliorer la prise de décision » (DataBusiness.fr, 2014). Par conséquent, en référence aux travaux d'Ève Chiapello et de Patrick Gilbert (2013), les TBDM sont des outils de management de l'innovation puisqu'une de leurs principales fonctions est de permettre aux entreprises de concevoir des innovations de produit/service et/ou des innovations organisationnelles plus adaptées et performantes sur le plan socioéconomique.

Afin de mieux comprendre dans quelle mesure les TBDM peuvent contribuer à améliorer les processus d'innovation de l'entreprise, nous proposons dans cet article de nous appuyer sur une enquête de terrain conduite de manière inductive (Glaser et Strauss, 1967) afin de répondre au questionnement suivant: comment les TBDM sont-ils intégrés au sein de l'entreprise et quels changements organisationnels impliquent-ils (section 2) ? Comment permettent-ils de développer des connaissances à la fois nouvelles et intéressantes pour l'organisation (section 3) ? Et finalement, à travers ces connaissances, comment permettent-ils d'améliorer les processus d'innovation de l'entreprise (section 4)?

Pour répondre à cet ensemble de questions, nous commençons par présenter le cadre théorique et la méthode d'enquête que nous avons mobilisés pour réaliser cet article (section 1). Nous montrons ensuite que les TBDM sont d'abord une innovation de produit sur le marché des technologies big data. Ainsi, nous verrons qu'afin d'être implémentés au sein de

\footnotetext{
2 À travers la notion de «re-présentation», Bruno Latour (1993) souhaite désigner la manière dont la connaissance scientifique s'opère par le biais d'un enchaînement de médiations qui ont pour fonction de présenter autrement l'objet observé. Ici, nous retrouvons donc la notion de «traduction » de la Sociologie des Sciences et de Techniques (Akrich et al., 2006) puisque chacune de ces médiations constitue une manière de réduire la réalité à un ensemble de caractéristiques bien spécifiques de façon à pouvoir l'amplifier à travers divers procédés de comparaison.
} 
l'entreprise, les TBDM impliquent un certain nombre de changements culturels et organisationnels. En ce sens, nous dégageons trois grandes difficultés caractérisant le processus d'intégration des TBDM au sein de l'entreprise et nous soulignons quelques cas de solutions déployées pour les dépasser (section 2). Nous pointons alors les changements cognitifs qui sont associés aux usages des TBDM. Et, nous montrons qu'en permettant à leur utilisateur d'exploiter de nouvelles stratégies d'exploration des données, les TBDM favorisent un rapport à l'enquête plus sérendipitaire ${ }^{3}$ (section 3 ). Finalement, nous verrons que les TBDM encouragent les stratégies d'exploration des usages qui sont de l'ordre de la percolation $^{4}$ et de l'internalisation (section 4). En conclusion, nous soulignons que, en tant qu'outils de management de l'innovation, si les TBDM peuvent permettre aux organisations d'améliorer la performance de leur processus d'innovation incrémentale, il est néanmoins possible de questionner leur apport dans le cas de processus d'innovation de rupture.

\section{CADRE THÉORIQUE ET MÉTHODOLOGIE D'ENQUÊTE}

Comme nous l'avons vu en amont, les TBDM sont des outils de gestion. À ce titre, d'un point de vue sociologique, ils peuvent être examinés à travers quatre grandes approches (Chiapello et Gilbert, 2013). La première est dite critique est consiste à repérer comment les outils de gestion exercent des formes de violences symboliques qui participent à instituer les états d'exploitation ( $c f$. Boussard, 2001 ; Dejours, 2003 ; Halpern et Le Galès, 2011). La deuxième est dite institutionnaliste est vise à comprendre le rôle des institutions/conventions dans la conception des outils de gestion ainsi que les effets de structure qui sont associés à leurs applications ( $c f$. Desrosières et Thévenot, 1988 ; DiMaggio et Powell, 1983 ; Orlikowski, 1992). La troisième est dite interactionnelle est cherche, d'une manière générale, à saisir pragmatiquement le processus d'inter-structuration qui se joue entre l'outil de gestion et les pratiques sociales ( $c f$. Barrey, 2007 ; Béguin et Rabardel, 2000 ; Fraenkel, 1995 ; Reynaud, 2001).

\footnotetext{
${ }^{3}$ La notion de sérendipité réfère à cette faculté humaine qui consiste à faire des trouvailles par sagacité accidentelle. Plus précisément, selon Robert K. Merton et Elinor G. Barber (2004), elle renvoie à un processus de découverte compris comme une séquence de trois états : celui de l'observation surprenante, celui de l'évaluation curieuse et celui de l'intérêt stratégique. Le raisonnement sérendipitaire est alors compris par opposition au raisonnement hypothético-déductif qui caractérise traditionnellement les activités de recherche scientifique : selon Merton et Barber (2004), les réelles découvertes découlent d'un processus de sérendipité.

${ }^{4}$ Alexandre Mallard (2011) parle de «percolation » afin de désigner une stratégie d'exploration des usages où les frontières entre utilisateur et concepteur sont très perméables. Comme nous le verrons par la suite, cette stratégie permet, entre autre, d'explorer les usages sans les avoir pour autant préconfiguré par diverses épreuves, et cela, durant l'ensemble des phases de conception et de commercialisation de l'innovation.
} 
Nos travaux s'inscrivent directement dans la troisième perspective dite interactionnelle. En ce sens, ils sont largement influencés par la théorie ancrée (i.e. : grounded theory) de Barney Glaser et d'Anselm Strauss (1967) et par la théorie de l'acteur-réseau de Madeleine Akrich, Michel Callon et Bruno Latour (2006). Durant notre enquête nous avons en effet cherché :

- à adopter une posture inductive consistant à accorder au terrain une place de premier choix ;

- à mieux saisir la manière dont les objets techniques que sont les TBDM associent les éléments humains et matériels qui composent l'organisation.

Avant de présenter nos terrains d'investigation, nous souhaitons préciser que nous avons anonymisé l'ensemble des acteurs économiques dont nous faisons référence dans ce texte ; c'est-à-dire, les noms des entreprises et les prénoms des professionnels présentés dans le tableau ci-dessous.

\begin{tabular}{|c|c|c|c|}
\hline Offreur de TBDM & Demandeur de TBDM & Partenaire & Autres \\
\hline $\begin{array}{l}\text { SoftwareDashboard: } \\
\text { (Landry, Sales } \\
\quad \text { Consultant) } \\
\text { ViewAnalytics: (Pierre- } \\
\text { André, Senior Solution } \\
\text { Architect) }\end{array}$ & $\begin{array}{c}\text { EasyAir: (aucun) } \\
\text { LabDerma: (Colline, } \\
\text { Responsable Market } \\
\text { Insights; Samuel, BI \& } \\
\text { Reporting Competence } \\
\text { Center Manager) }\end{array}$ & $\begin{array}{c}\text { Sixty-six: (Héloïse, Data } \\
\text { analyst) } \\
\text { SITIC France: } \\
\text { (Capucine, Responsable } \\
\text { Avant-vente Solutions } \\
\text { Digitales) }\end{array}$ & $\begin{array}{l}\text { DecIT: (Gislain, } \\
\text { Fondateur du magazine } \\
\text { spécialisé dans } \\
\text { l'informatique } \\
\text { décisionnel) }\end{array}$ \\
\hline $\begin{array}{l}\text { Secass France : (aucun) } \\
\text { PisaTIC: (Justin, } \\
\text { Responsable de l'offre } \\
\text { PisaTIC Business } \\
\text { Analytics) }\end{array}$ & $\begin{array}{c}\text { CarBlue : (Marin, } \\
\text { Responsable Offre CRM) } \\
\text { SRP : (Calvin, } \\
\text { Responsable Étude et } \\
\text { Stratégie) }\end{array}$ & Genicap : (Gabriel, PDG) & \\
\hline $\begin{array}{l}\text { WT Quintet: (Odelia, } \\
\text { Sales Excutive) }\end{array}$ & $\begin{array}{l}\text { Hotes de Voyage: (Peter, } \\
\text { Directeur Commercial } \\
\text { Opérations \& e-business) }\end{array}$ & & \\
\hline
\end{tabular}

Figure 1 : Tableau 1

Aussi, nos terrains d'investigation sont les suivants :

- le salon Big Data 2014 qui a regroupé plus de 80 exposants ;

- le salon E-Marketing et Stratégie Client 2014 qui a regroupé plus de 350 exposants ;

- le ViewAnalytics Tour organisé par ViewAnalytics en 2013 ;

- le SoftwareDashboard Tour organisé par SoftwareDashboard en 2014.

Au cours de ces quatre terrains, nous avons pu recueillir différents matériaux qui ont à chaque fois été analysés à travers un traitement spécifique : 
- Une série de journaux spécialisés traitant de la thématique du big data et une série de documentations présentant les technologies d'un certain nombre d'exposants présents sur les salons. Ces documents ont fait l'objet d'une lecture et d'une analyse exploratoire.

- Une série d'enregistrements audio de 20 présentations réalisées par des offreurs de technologies big data. Chaque présentation consistait à exposer les caractéristiques techniques des solutions développées par les offreurs et à présenter quelques cas d'usage. Ces présentations d'une durée moyenne de 50 minutes ont systématiquement fait l'objet d'une prise de note détaillée, d'une retranscription partielle et d'une analyse focalisée sur les usages des TBDM.

- Une série d'enregistrements audio de 11 retours d'expérience réalisés par des demandeurs de technologies big data. Chaque retour d'expérience consistait cette foisci à présenter le processus d'intégration des technologies big data au sein de l'entreprise, à pointer les difficultés/facilités rencontrées durant ce processus et à exposer les avantages/inconvénients associés aux usages des big data. Ces retours d'expérience d'une durée moyenne de 50 minutes ont également fait l'objet d'une prise de note détaillée, d'une retranscription partielle et d'une analyse centrée sur les usages des TBDM.

- Une série d'enregistrements vidéo de 4 démonstrations d'utilisation des TBDM réalisées par des offreurs-utilisateurs de ces technologies. Ces enregistrements d'une durée moyenne de 15 minutes ont fait l'objet d'une description et d'une analyse détaillée des activités présentées.

Notons que l'ensemble de ces matériaux ont un statut particulier puisqu'ils ont à chaque fois été produits dans le cadre d'une stratégie de communication. Cependant, il est important de rappeler que ces stratégies de communication ont systématiquement été orientées vers un public de professionnels dont certains sont des experts du big data. Par conséquent, s'il ressort que nos matériaux portent la trace des logiques commerciales des offreurs, ils n'en restent pas moins pertinents compte tenu que les discours dont ils témoignent sont construits par des professionnels du big data et pour des professionnels souhaitant adopter/améliorer les/leurs technologies big data. À la différence des entretiens qui sont généralement produits à l'intérieur d'un cadre d'interaction relativement libre, nos matériaux bénéficient et pâtissent donc des avantages et des inconvénients d'un discours commercial qui est plus ou moins implicitement régulé par le corps des professionnels du big data. 
En conclusion, une fois compris dans leur ensemble, nos matériaux véhiculent un ensemble de représentations et de pratiques qui offrent un point de vue intéressant pour examiner les usages que les entreprises font des TBDM.

\section{UN PROJET D'INNOVATION SOCIOTECHNIQUE}

Le premier constat que nous avons pu faire durant notre enquête est que l'adoption des TBDM constitue pour l'entreprise un projet d'innovation sociotechnique qui sous-tend des changements importants sur le plan organisationnel.

Afin de bien saisir les formes de ces changements, nous montrons dans la section 2.1 que les TBDM constituent avant tout un marché. Nous soulignons que, pour l'entreprise (i.e. : le demandeur), la première difficulté consiste à établir des passerelles communicationnelles entre la Direction des Services Informatiques (DSI) et les Services de Gestion (SG), et à désigner les porteurs du projet. Nous verrons qu'une fois cette collaboration établie et les porteurs du projet désignés, ces derniers doivent enrôler les unités opérationnelles concernées par l'intégration des TBDM (section 2.2). Ensuite, nous pointons la dernière grande difficulté pour les porteurs du projet : enrôler les unités décisionnelles de l'entreprise de façon à les conduire à adopter les TBDM ou, a minima, à accepter les formats de communication qui y sont associés (section 2.3).

\subsection{Du marché des TBDM à la collaboration entre la DSI et les SG}

Comme nous l'avons souligné, il est important de rappeler qu'avant d'être une potentielle source d'avantage concurrentiel pour les demandeurs, les TBDM sont générateurs de profit pour les offreurs. Autrement dit, ils constituent une innovation de produit qui forme un marché autour duquel peuvent graviter jusqu'à trois types d'acteurs :

- l'entreprise qui souhaite adopter les TBDM (i.e. : le demandeur) ;

- l'entreprise qui développe et commercialise les TBDM, voire, qui accompagne parfois leur implémentation au sein de l'organisation (i.e. : l'offreur) ;

- l'entreprise de conseils qui, dans certain cas et en collaboration avec l'offreur, prend en charge la phase d'accompagnement du demandeur (i.e. : le partenaire).

C'est ainsi que Sixty-Six, en partenariat avec SoftwareDashboard (i.e. : l'offreur), a par exemple assisté EasyAir (i.e. : le demandeur) afin de leur permettre de piloter plus facilement leur achat média en cartographiant l'hésitation de ses visiteurs sur Internet; ou encore, que 
ViewAnalytics (i.e.: l'offreur) a directement suivi LabDerma (i.e : le demandeur) afin d'améliorer la réactivité de son système décisionnel.

Du point de vue du demandeur, la première grande difficulté est alors d'instaurer de nouvelles passerelles communicationnelles entre la DSI et les SG, et de désigner des porteurs de projet capables de croiser les compétences techniques et organisationnelles de l'entreprise.

«Vous avez besoin d'apprendre le langage de l'autre. Côté métier [i.e. : les SG], côté IT [i.e. : la DSI], il faut se comprendre. Il faut mettre un décodeur parfois ! Mais heureusement, avec Samuel ${ }^{5}$, on a réussi à trouver un langage commun. » (Retour d'expérience, Colline, Responsable Market Insights, Laboratoire pharmaceutique LabDerma, 2014).

En effet, les TBDM ont pour finalité de redistribuer vers les unités opérationnelles et décisionnelles de l'entreprise une partie du travail afférant traditionnellement à la DSI ${ }^{6}$. Et, la réussite de l'adoption des TBDM au sein de l'entreprise dépend principalement des formes de cette redistribution et de son acceptation par l'ensemble des employés concernés par ce projet. Il est donc important de soigner la bonne intégration humaine et sociale (et pas seulement technique) des TBDM afin d'éviter leur rejet par l'organisation.

«Le challenge principal, le défi le plus important qui a été rencontré par les entreprises, il n'est pas technologique; il est organisationnel. Le gros challenge aujourd'hui, c'est la culture d'entreprise et c'est l'organisation dans l'entreprise. Comment je fais pour réussir à faire passer l'entreprise en mode data driven [i.e. : gouvernance par la donnée] ?» (Retour d'expérience, Capucine, Responsable Avant-vente Solutions Digitales, société de services informatiques SITIC France, 2014).

La DSI ne peut donc pas, à elle seule, assurer l'implémentation sociotechnique des TBDM : elle doit nécessairement collaborer avec les SG afin de pouvoir lever les défis technologiques mais aussi, et surtout, organisationnels qui y sont associés. Ce point est d'autant plus

\footnotetext{
${ }_{6}^{5}$ Samuel, BI \& Reporting Competence Center Manager, laboratoire pharmaceutique LabDerma.

${ }^{6}$ Notons que ce phénomène n'est pas nous nouveau et n'est pas propre aux TBDM puisque, dès 2000, avec le développement de l'Intranet dans les entreprises (Loisel, 2000) ou encore, plus récemment, avec l'urbanisation des systèmes d'information dans les organisations, il s'agit également de redistribuer le travail réalisé par la DSI. Cependant, et nous y reviendrons plus en détail par la suite, l'implémentation des TBDM au sein de l'entreprise conduit à une modification importante de la répartition du travail de traitement et d'analyse des données de l'entreprise. Ce qui implique alors un partage des compétences en informatique et en statistique au sein de l'organisation qui est assez nouveau. Et, les TBDM favorisent justement ce partage puisqu'ils constituent des outils de traitement et d'analyse simplifiant considérablement les activités de navigation au sein de gigantesques bases de données.
} 
considérable qu'une fois cette collaboration établie, les porteurs du projet doivent enrôler les unités opérationnelles susceptibles d'utiliser les TBDM.

\subsection{Enrôler les unités opérationnelles de l'entreprise}

La deuxième grande difficulté associée au projet d'adoption des TBDM consiste alors à définir clairement les métiers des unités opérationnelles qui doivent utiliser ces technologies afin de déterminer leur besoin informationnel.

Pour ce faire, Samuel et Colline ont par exemple été conduits à recueillir l'ensemble des documents quotidiennement mobilisés par les forces de ventes des 33 filiales de LabDerma afin d'en faire une synthèse et ainsi développer une application mobile destinée à répondre aux besoins d'information de leurs équipes commerciales. De façon quasi-identique, Marin de l'entreprise Carblue a pu mettre en place, avec la société Secass France, une application des TBDM destinée à améliorer la visibilité que ses équipes commerciales ont de leur parc automobile. De cette façon, aujourd'hui, lorsque qu'un commercial de l'entreprise Carblue arrive sur la page d'accueil de cette application :

«Il voit des histogrammes et des compteurs et tout de suite ça lui va bien : il a une synthèse sur son parc. [...] Il connait la part de Carblue, [...] en fonction des couleurs des histogrammes, il a une analyse qui lui est faite. Spontanément, il peut savoir qui est le concurrent qui est le plus virulent sur son secteur. Si je prends ce cas précis, [...] sur ce segment là du marché, j’ai quelque chose à faire face à Redcar [...]. Alors, on passe à l'étape suivante, et là, on a un tableau par segment [...]. Vous avez le nombre de véhicules concernés, ce sont toutes les immatriculations des véhicules associés qu'il y a sur ce territoire, et vous avez, en bout de liste, des véhicules. Vous cliquez sur «liste des véhicules » et tous les véhicules du segment apparaissent. Vous avez alors la possibilité de savoir qui est votre concurrent et chez qui il vous pénalise. » (Retour d'expérience, Marin, Responsable Offre CRM, concessionnaire automobile Carblue, 2014).

Calvin, Responsable Étude et Stratégie du site de e-commerce SRP, souligne alors l'importance de co-élaborer avec les unités opérationnelles une définition assez large de leur besoin informationnel afin de leur permettre de réaliser, dans un périmètre bien entendu préalablement circonscrit, des activités d'exploration des données. De ce fait, les unités opérationnelles ont la possibilité de participer à la construction des connaissances de 
l'entreprise en dégageant des associations de données qui peuvent être intéressantes compte tenu de leur caractère souvent très pragmatique et de leur ancrage empirique.

Par conséquent, cette phase d'enrôlement est fondamentale puisqu'elle participe à définir les formes de l'implication des unités opérationnelles dans ce que Gilbert de Terssac nomme le travail d'organisation (2002).

\subsection{Enrôler les unités décisionnelles de l'entreprise}

Finalement, les porteurs du projet doivent également enrôler les unités décisionnelles de l'entreprise. Ainsi, la troisième grande difficulté est, cette fois-ci, d'amener les directions à modifier leurs habitudes de travail en les conduisant à faire usage des TBDM. Précisons que cette tâche n'est pas des moindres compte tenu du fait que l'implémentation de ces technologies implique une modification de la culture décisionnelle de l'entreprise.

«Le dashboarding [i.e. : l'activité de conception et d'utilisation des tableaux de bord] est une philosophie qu'il faut savoir appréhender. Ce n'est pas du reporting [i.e. : l'activité de conception et d'utilisation des rapports]. Nous, on a mis un peu de temps à digérer la transition reporting-dashboarding. Il ne faut pas la sousestimer. [...] Le problème, c'est vraiment la transition du PowerPoint statique à la configuration du dashboard dynamique. [...] Chez nous, les gens étaient habitués à recevoir des tableaux Excel et ils refaisaient le monde. Là, les tableaux de données, maintenant, c'est eux qui les font ; c'est eux qui analysent. C'était un changement culturel vraiment important pour nous.»(Retour d'expérience, Samuel, BI \& Reporting Competence Center Manager, laboratoire pharmaceutique LabDerma, 2014)

« Notre objectif 2014-2015, c'est de faire en sorte de faire passer nos rapports $P d f$, qui sont tout de même assez lourds à construire, par des tableaux un peu plus maniables directement dans ViewAnalytics. » (Retour d'expérience, Colline, Responsable Market Insights, laboratoire pharmaceutique LabDerma, 2014)

Les deux verbatims exposés ci-dessus illustrent bien le problème que pose l'enrôlement des unités décisionnelles dans un projet d'adoption des TBDM. De manières traditionnelles, les directions ont l'habitude de prendre leur décision en fonction des rapports d'activités et/ou des rapports d'études de marché élaborés par les managers de l'entreprise. Aussi, ces rapports sont habituellement élaborés et communiqués à travers le triptyque Excel-PowerPoint-Pdf qui sous-tend un usage de l'information plutôt statique et non-interactif. En outre, les activités de 
conception des rapports s'inscrivent généralement dans des temporalités plutôt longues compte tenu que l'accès aux données est traditionnellement intermédié par la DSI.

Or, comme nous le verrons par la suite, avec les TBDM, les managers ont un accès privilégié aux données de l'entreprise : ils ne sont plus contraints de formaliser une demande à la DSI. En outre, les formats de communication associés aux TBDM sont dynamiques: les destinataires des documents, tout comme leurs producteurs, peuvent manipuler, par exemple lors d'une réunion et en temps réel, les différentes dimensions intégrées au tableau de bord.

Dès lors, étant donné que les TBDM constituent un changement matériel et culturel considérable, il est important de souligner que leur diffusion au sein des directions n'est pas toujours aisée.

« Tout le monde n'a pas encore passé le pas. On a déjà une bonne utilisation mais ça prend du temps. C'est normal, c'est de l'éducation et c'est de la communication. » (Retour d'expérience, Colline, Responsable Market Insights, laboratoire pharmaceutique LabDerma, 2014)

Néanmoins, les grands décideurs de l'entreprise semblent assez curieux voire intéressés par les TBDM.

«On a remarqué par les logs que les présidents se connectent à l'outil. Demander à un président et un vice président de se connecter à un outil, c'est parfois un challenge! On a remarqué qu'ils s'y connectent, donc, ils s'y retrouvent. » (Retour d'expérience, Samuel, BI \& Reporting Competence Center Manager, laboratoire pharmaceutique LabDerma, 2014)

\section{EXPLOITER DE NOUVELLES STRATÉGIES D'EXPLORATION DES DONNÉES}

L'implémentation des TBDM au sein de l'entreprise est un projet d'innovation sociotechnique. Plus précisément, l'adoption par l'entreprise de ces technologies implique une restructuration plus ou moins importante de l'organisation de façon à garantir leur bonne diffusion et leur bonne acceptation. Car, comme nous l'avons exposé dans la section précédente, les TBDM recouvrent une architecture informationnelle particulière qui, une fois déployée au sein de l'organisation, permet d'instaurer de nouveaux attachements entre les unités opérationnelles et décisionnelles qui la composent.

Dans cette deuxième section, nous souhaitons ajouter que les TBDM permettent également d'instituer de nouveaux attachements entre l'entreprise et son environnement : en fluidifiant la 
circulation des données internes et externes à l'organisation, les TBDM favorisent l'exploitation, par leur utilisateur, de nouvelles stratégies d'exploration des données (March, 1991 ; Mothe et Brion, 2008). En ce sens, nous montrons ici que les TBDM composent des sortes de nœuds d'informations qui, en augmentant et facilitant la diversité des possibilités de croisement des données, permettent des processus de construction de connaissances plus sérendipitaires (Merton et Barber, 2004).

Pour rendre compte de cet aspect, nous commençons par pointer comment, à travers les technologies big data, les TBDM facilitent le travail de recueil et de traitement des données. Nous verrons ainsi que les TBDM permettent de lever une certaine inertie organisationnelle qui caractérise traditionnellement les activités d'enquête au sein de l'entreprise (section 3.1). Ensuite, nous présentons comment l'ergonomie logicielle des TBDM participe à instituer un rapport à l'enquête plus exploratoire (section 3.2). Dans la section 3.3, nous verrons alors que les TBDM contribuent finalement à instaurer des processus de construction des connaissances plus flexibles et collaboratifs.

\subsection{Les TBDM facilitent le recueil et le traitement des données}

Nous avons vu dans la section 1 que les TBDM ont pour finalité de redistribuer, vers les unités opérationnelles et décisionnelles de l'entreprise, les activités de traitement et/ou d'analyse des données qui affèrent traditionnellement à la DSI. Du point de vue des managers et des rapports qu'ils entretiennent avec leur terrain d'investigation, les TBDM impliquent ainsi un changement important. Ce changement peut être compris à deux niveaux.

Le premier concerne le recueil des données. Car, comme nous l'avons mentionné en introduction, avec le développement toujours croissant de l'automation industrielle (Bernard, 2012 ; Naville, 1961), de l'électronisation du commerce (Rallet, 2001) et de celle de la production des biens et des services (Riccio et Bonnet, 2011), les consommateurs et les travailleurs sont conduits à produire une grande quantité de données numériques durant leurs activités quotidiennes (Kessous, 2012). Aussi, ces données ne sont pas sollicitées puisque les processus de leur captation et de leur stockage s'opèrent principalement de façon nondéclarative, continue et automatique. Par conséquent, avec les TBDM, les managers n'ont plus besoin de conduire une étude pour produire des informations (Vayre, 2013a). D'une certaine façon, ce sont donc les phases de problématisation, de constitution du dispositif méthodologique et du recueil des données qui s'en trouvent considérablement allégées de telle sorte que les temporalités de l'enquête sont remarquablement raccourcies. 
Ce qui nous conduit au deuxième niveau de changement qui est celui du traitement des données. En effet, les facilités que nous venons de souligner sont en quelque sorte amplifiées par la nouvelle division du travail que permet l'implémentation des TBDM. Rappelons qu'avec les TBDM, la DSI n'a plus le monopôle du traitement des données de l'entreprise : les managers n'ont donc plus besoin d'effectuer une demande auprès de la DSI pour accéder aux données de l'entreprise.

«Avant les gens étaient très sages, j'avais une société, mes données étaient à l'intérieur de la société, elles étaient entreposées par la DSI, on demandait à la DSI des rapports et ils nous fournissaient les rapports. Maintenant, il y a une espèce d'émancipation des utilisateurs qui peuvent à la fois traiter de façon beaucoup plus simple et beaucoup plus rapide les données de l'entreprise. » (Présentation, Justin, Responsable de l'offre PisaTIC Business Analytics, éditeur de logiciels décisionnels PisaTIC France, 2014)

Du point de vue des managers, ce point est particulièrement important puisque les TBDM leur permettent ainsi d'éviter la phase de formalisation de la demande et celle de son traitement auprès de/par la DSI. La métaphore de la bicyclette mobilisée ci-dessous illustre bien cet aspect.

«Quand je me ballade dans la rue et que je cherche une adresse, je prends mon iPhone, je tape dans Google Map ou le Map d'Apple, et puis en quelques clics je vais pouvoir trouver le restaurant ou le magasin que je cherchais [...]. Donc rapidement, j'ai une bicyclette à mon esprit. C'est facile de faire [...] ce genre de démarche. Quand je suis dans une entreprise et que j'ai besoin de faire un tableau de bord [...] pour identifier les 3 produits qui présentent la marge la plus négative sur un environnement important d'information, qu'est-ce que je fais ? Je lance une demande auprès de la DSI qui lance un processus pour mettre en place un rapport au travers d'un traitement d'un univers $x$ ou $y$. Je n'ai donc pas cette possibilité d'avoir une bicyclette à mon esprit. » (Présentation, Landry, Sales Consultant, éditeur de logiciels décisionnels SoftwareDashboard, 2014)

Et, comme nous l'exposons dans la section suivante, les TBDM constituent effectivement cette sorte de bicyclette cognitive permettant au manager d'effectuer l'enquête de façon plus flexible et intuitive. Car, en référence à la section 1.3, l'architecture logicielle des TBDM vient faciliter les investigations des managers en leur permettant de dépasser les rigidités analytiques et communicationnelles imposées par le triptyque Excel-PowerPoint-Pdf. 
«Avant SoftwareDashboard, le reporting chez Sixty-Six, c'était le bon vieux Excel. Donc, par exemple, si on venait sur du reporting hebdomadaire on était susceptible de passer 5 heures par semaine à actualiser sa macro, vérifier que tout se passe bien et mettre ça en forme de manière pas trop moche. Aujourd'hui, avec SoftwareDashboard c'est vraiment plus simple puisque, certes, on a un premier set up qui est un petit peu plus lourd [...], environ deux jours pour un dashboard, mais après, l'actualisation, c'est automatique et tout se passe très bien. » (Retour d'expérience, Héloïse, Data analyst, agence de marketing digital Sixty-Six, 2014)

De ce fait, en modifiant les cadres relationnels et temporels de l'enquête, les TBDM permettent de lever une certaine inertie organisationnelle qui caractérise traditionnellement les activités de conception et de communication des rapports au sein de l'entreprise.

\subsection{Les TBDM instaurent un rapport à l'enquête plus exploratoire}

Nous souhaitons alors ajouter que ces facilitations des activités d'enquête sont prolongées par une ergonomie logicielle favorisant des formes de traitement de données plus exploratoires.

Pour ce faire, nous proposons d'examiner une partie de la démonstration d'usage réalisée par Pierre-André, Senior Solution Architect chez l'éditeur de logiciels décisionnels ViewAnalytics. Précisons que la séquence que nous avons sélectionnée s'insère dans le contexte d'activité suivant : Pierre-André vient de réaliser un premier parcours informationnel sur le tableau de bord de ViewAnalytics afin de repérer l'impact d'une des campagnes marketing que ViewAnalytics a réalisé sur Twitter. Il commence alors un nouveau parcours d'information. Afin de rendre compte du caractère exploratoire de ce deuxième parcours informationnel, la synthèse que nous proposons d'en faire marque une distinction nette entre les verbes trouver et découvrir :

- trouver une information implique nécessairement d'avoir préalablement formulé une question plus ou moins explicite et ainsi d'avoir préalablement conçu et conduit une stratégie de recherche d'information ;

- a contrario, découvrir une information n'implique aucune forme de questionnement dans le sens ou le chercheur ne peut découvrir une information qu'à condition de ne pas l'avoir préalablement recherchée.

Notons que chaque photographie exposée ci-dessous est généralement marquée par un cercle qui indique les actions de navigation, et/ou par une ellipse en pointillées qui indique les centres d'attention, et/ou par un carré qui indique les points d'interactivités. 
Partant, Pierre-André commence sans vraiment avoir d'objectif: après avoir observé rapidement les activités réalisées sur Twitter, il souhaite simplement regarder ce qui ce passe sur les autres canaux ( $c f$. photographie 1). Alors, il découvre un pic sur le graphique de YouTube (cf. photographie 2).

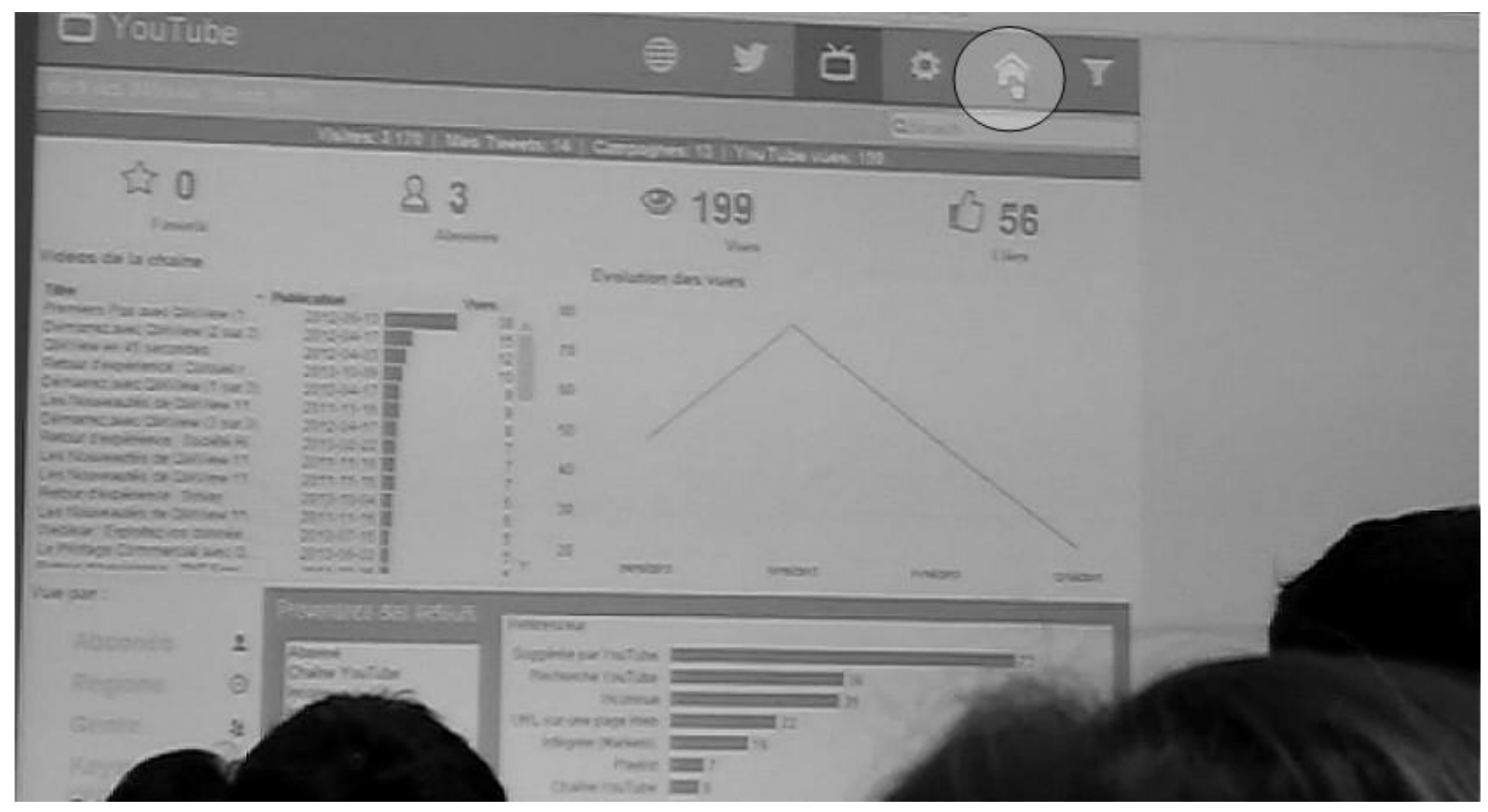

Figure 2 : Photographie 1

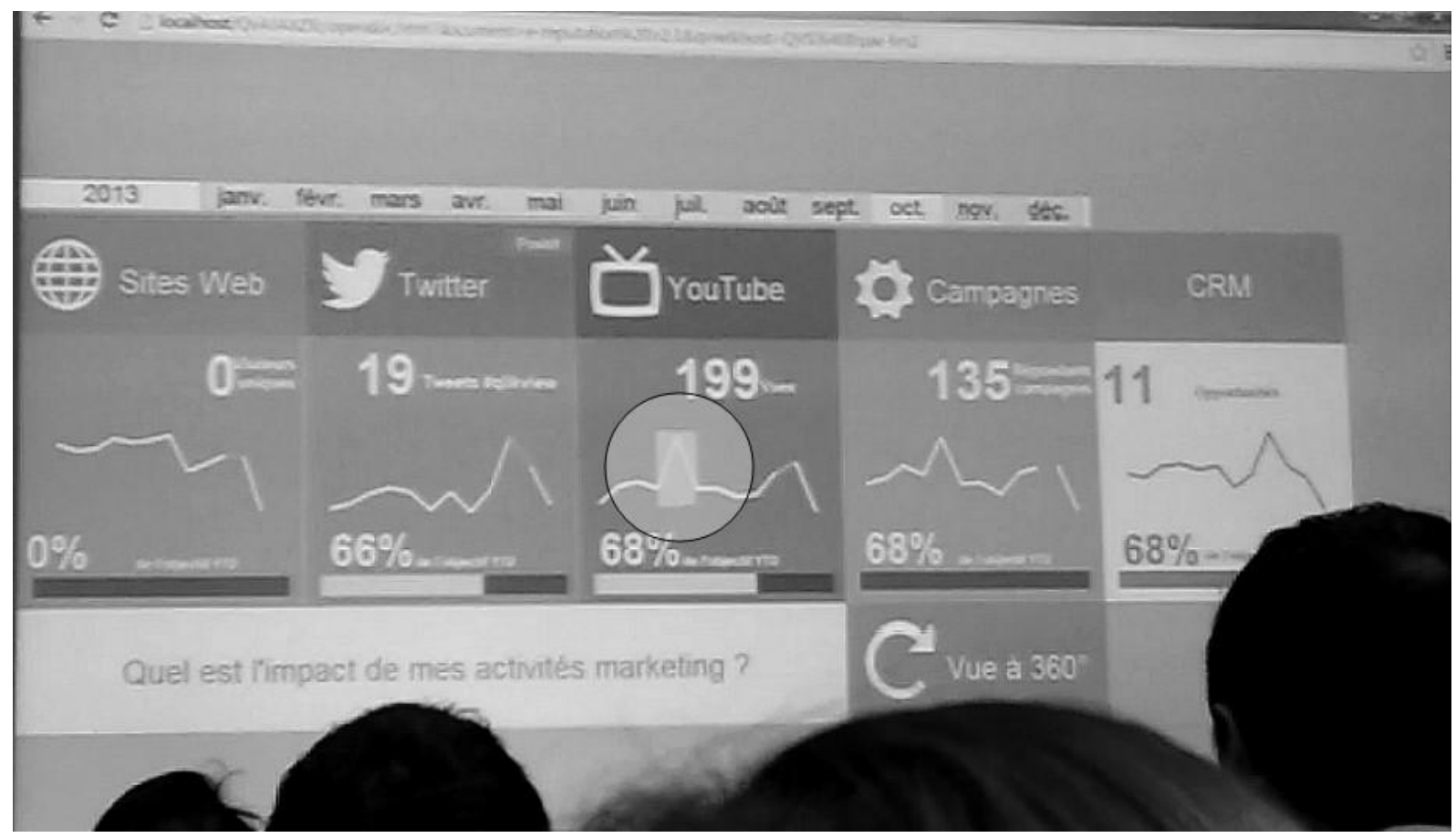

Figure 3 : Photographie 2 
Il finit alors par trouver une corrélation entre les Vues YouTube et les Répondants (cf. photographie 3).

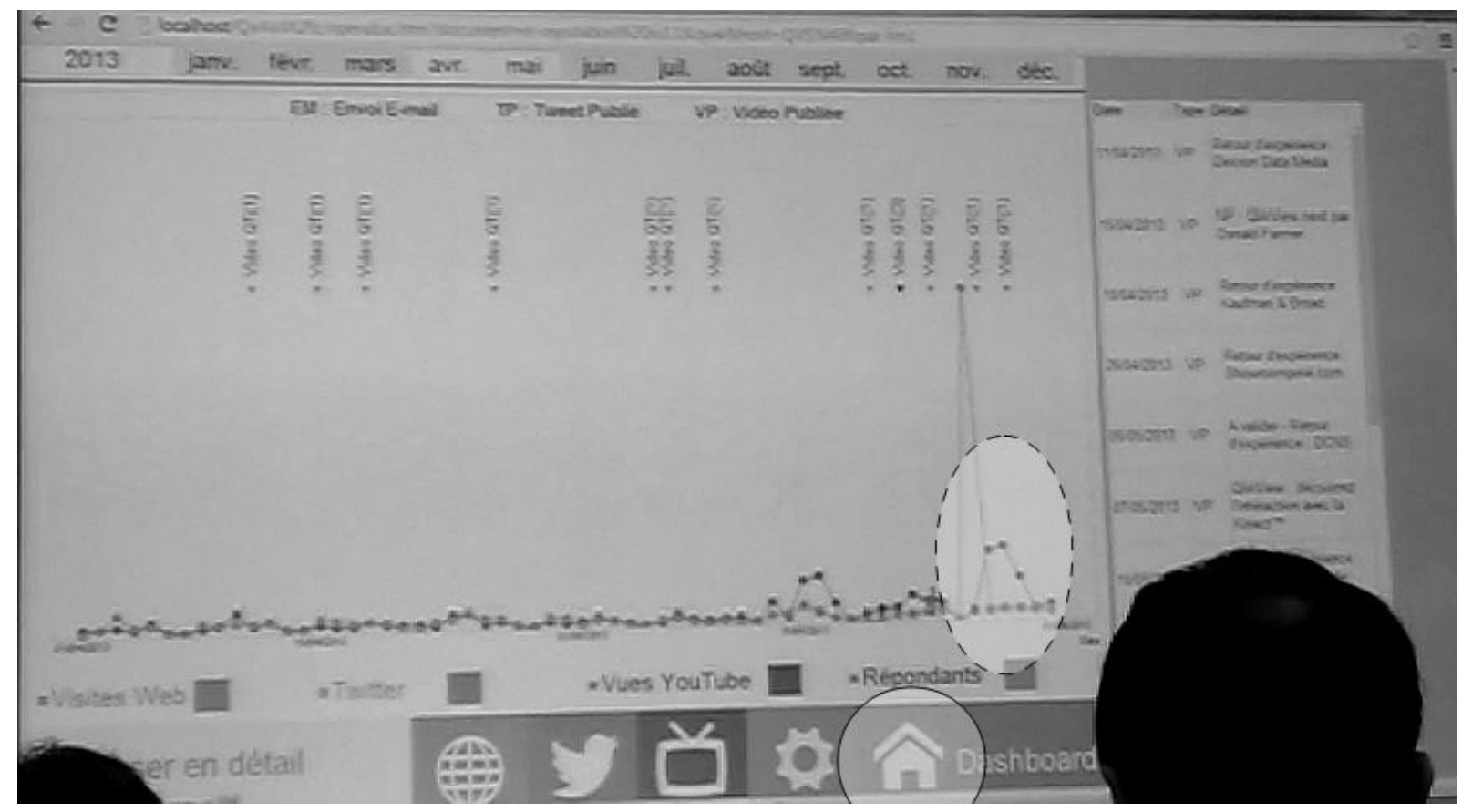

Figure 4 : Photographie 3

Puis, il découvre que les vidéos ViewAnalytics Set and Match sont la cause de cette corrélation ( $c f$. photographie 4).

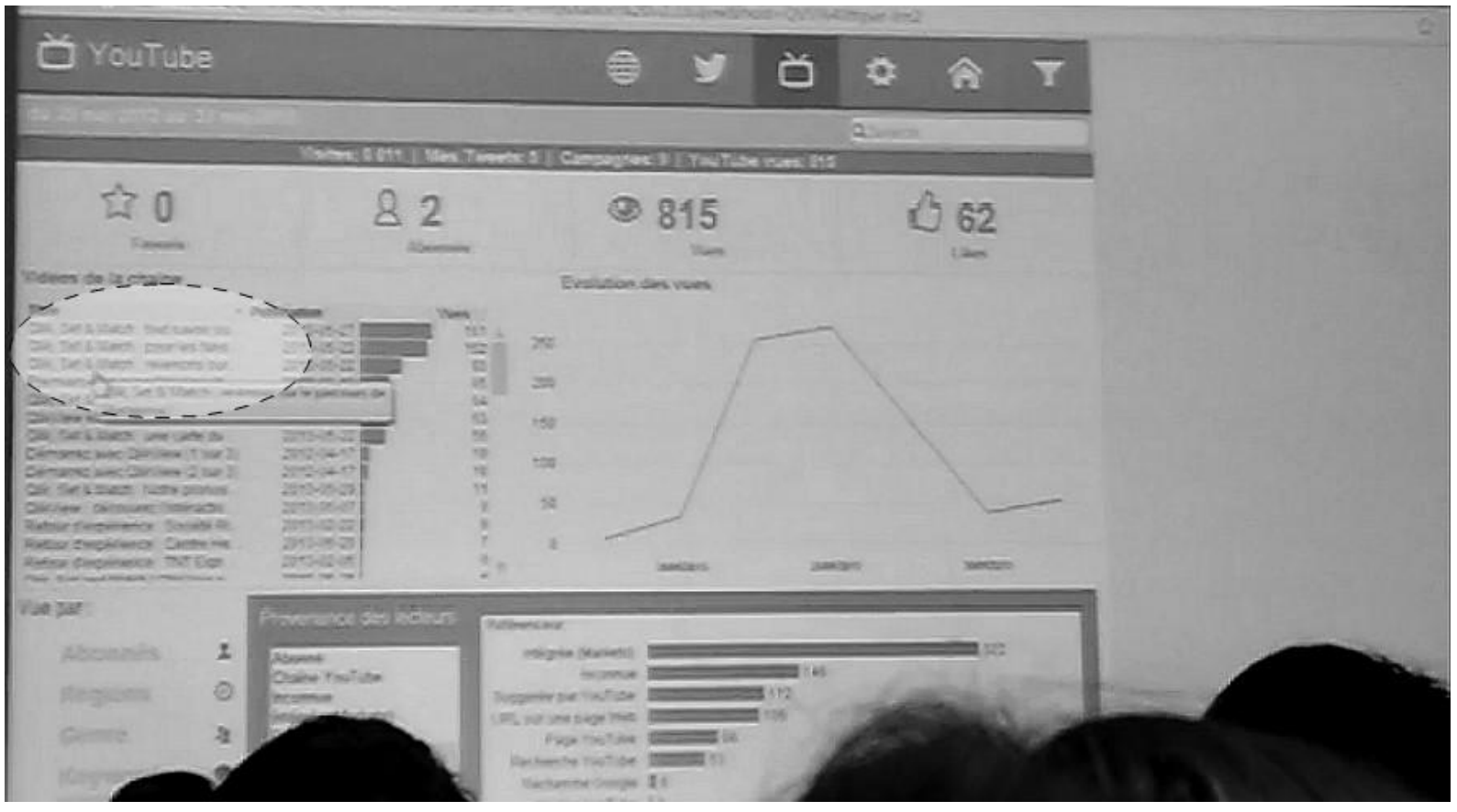

Figure 5 : Photographie 4 
Ensuite, il trouve que les lecteurs de ces vidéos viennent de Marketino ( $c f$. photographie 5) et qu'il y a bien une campagne de vidéos ViewAnalytics Set and Match qui a été conduite sur la période du pic observé ( $c f$. photographie 6).

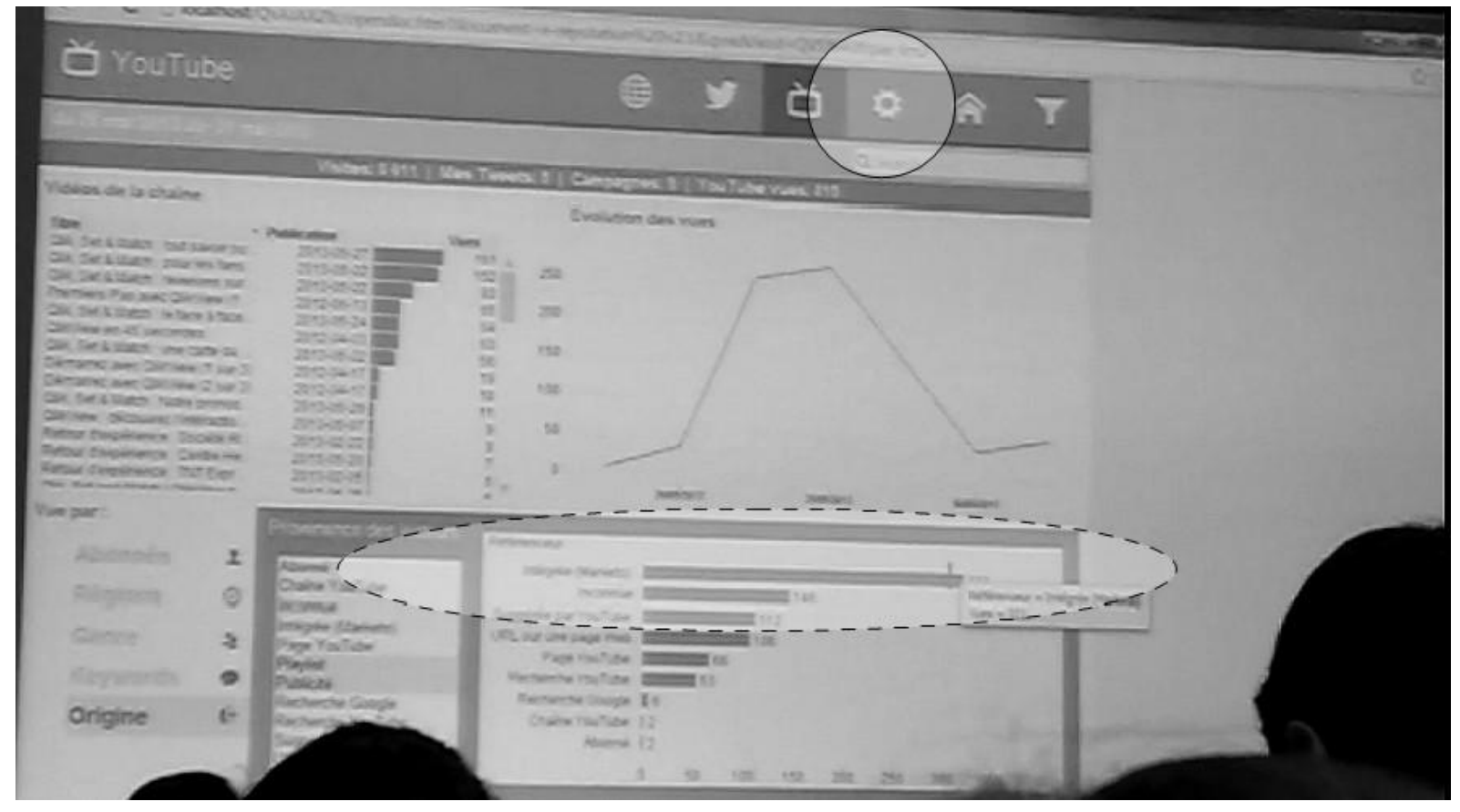

Figure 6 : Photographie 5

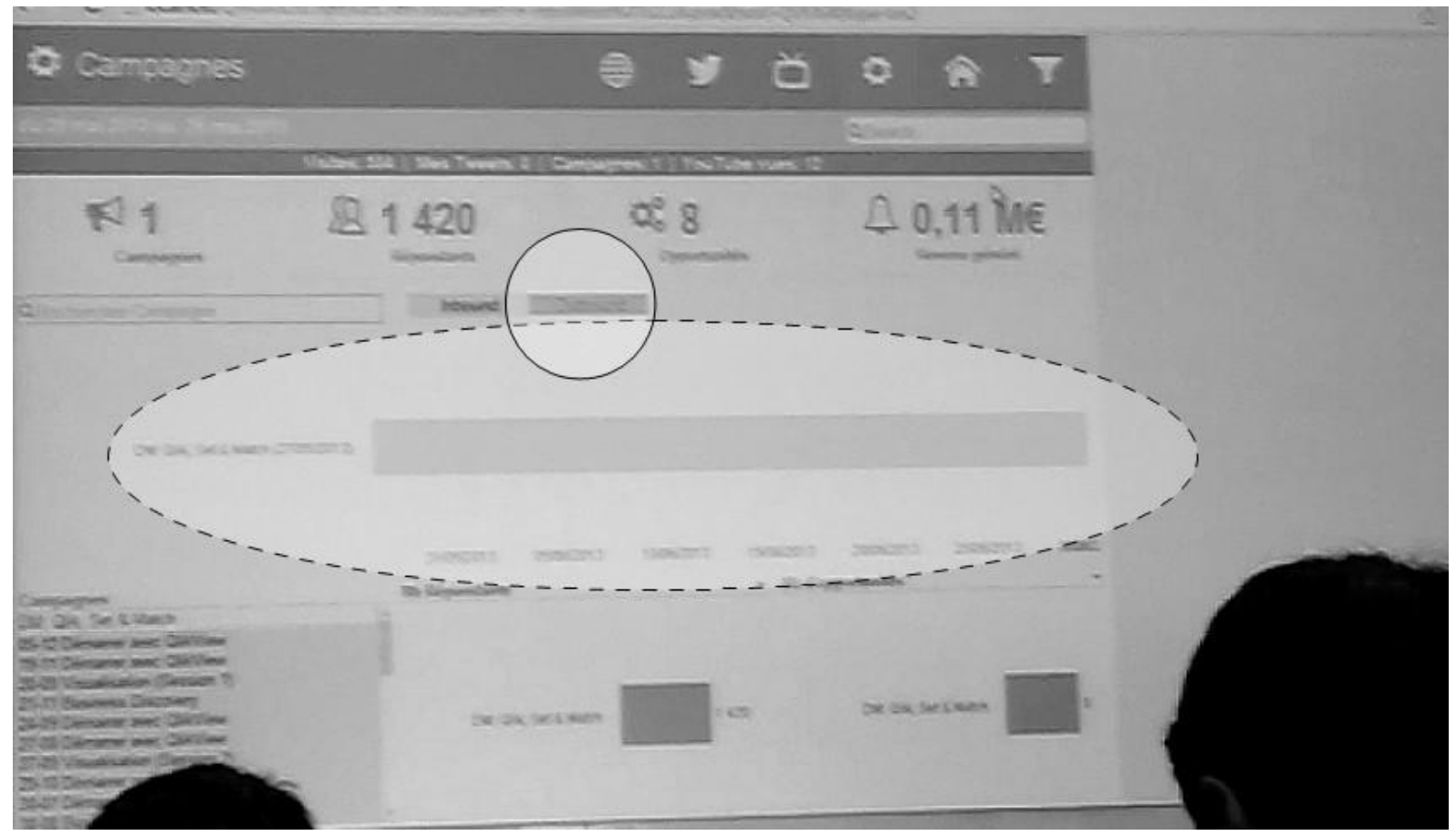

Figure 7 : Photographie 6 
Finalement, il découvre que cette campagne a généré du chiffre d'affaire ( $c f$. photographie 7) et il trouve qu'elle a également eu un impact sur Twitter ( $c f$. photographie 8).

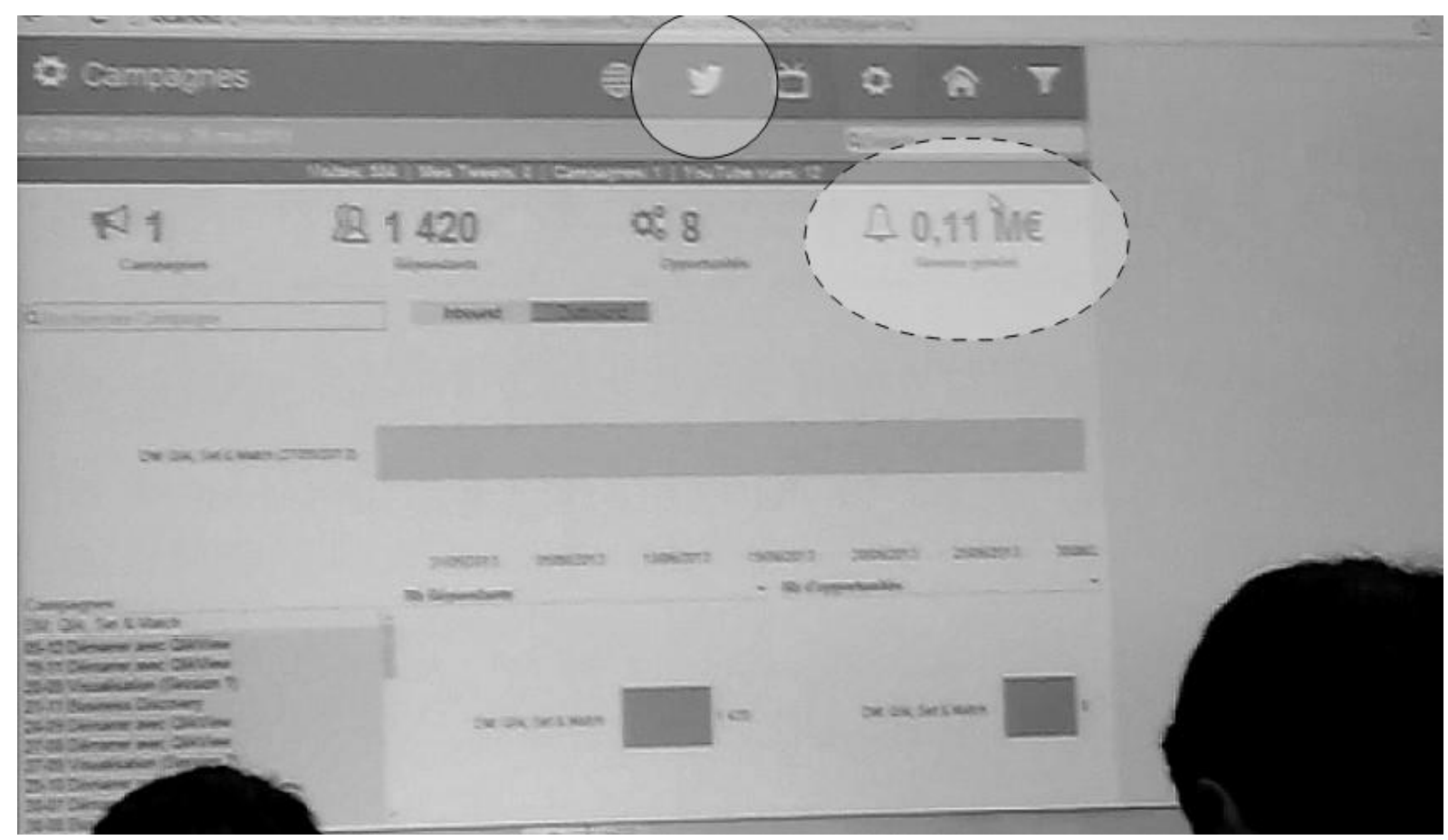

Figure 8 : Photographie 7

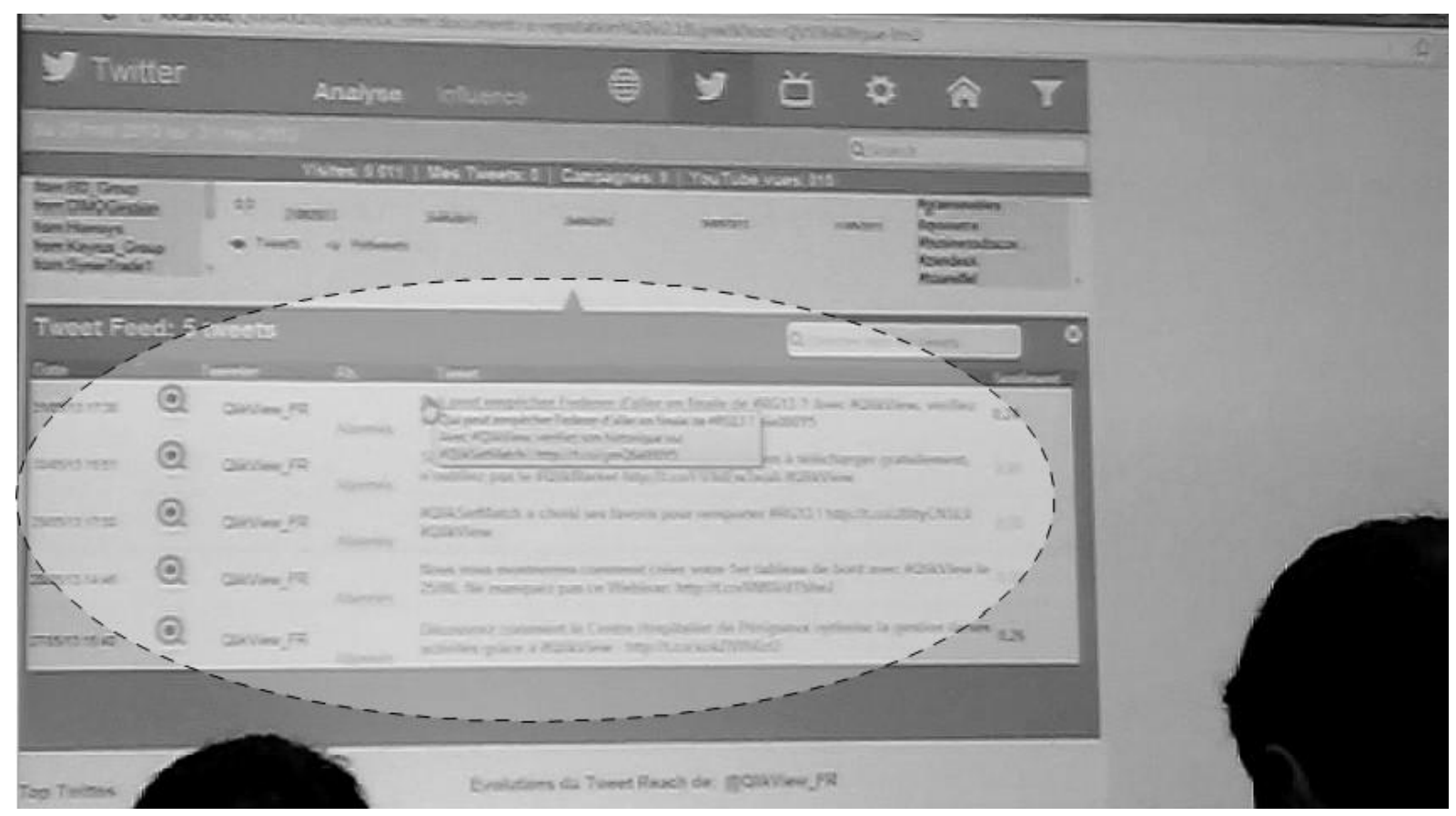

Figure 9 : Photographie 8 
Cet extrait de démonstration d'usage est intéressant puisqu'il illustre bien comment PierreAndré remarque que le pic YouTube est corrélé à la courbe des répondants et comment, à travers l'identification des vidéos ViewAnalytics Set and Match, il découvre progressivement que cette corrélation est la conséquence d'une de ses campagnes marketing. En d'autres termes, à la manière dont l'architecture physique peut favoriser l'innovation (Allen et Henn, 2007), le système de sélection par pic, la structure des liens facilitant le déplacement entre les différents tableaux, et celle plus globale du tableau de bord de ViewAnalytics, qui part d'une vue générale vers différentes vues de détails, encouragent les logiques d'exploration.

\subsection{Les TBDM suscitent un nouveau rapport à la construction des connaissances}

Plus précisément, les TBDM favorisent cette forme d'inférence bien particulière qu'est l'abduction (Peirce, 1974).

Rappelons que la déduction consiste à conclure un résultat (e.g. : cet oiseau est un cygne) à partir d'une règle (e.g. : les oiseaux blancs sont des cygnes) et d'un cas (e.g. : cet oiseau est blanc); et, que l'induction consiste à généraliser une règle (e.g. : les oiseaux blancs sont des cygnes) à partir d'un cas (e.g. : cet oiseau est blanc) et d'un résultat (e.g. : cet oiseau est un cygne). L'abduction se distingue ainsi de ces deux types d'inférences puisqu'elle consiste cette fois-ci à retracer un cas (e.g. : cet oiseau est blanc) à partir d'une règle (e.g. : les oiseaux blancs sont des cygnes) et d'un résultat (e.g. : cet oiseau est un cygne; Peirce, 1974). Autrement dit, à la différence de la déduction et de l'induction qui ont pour fonction respective d'appliquer ou de généraliser une règle à (partir d')un ensemble de cas, l'abduction à pour fonction de retracer l'explication d'un cas particulier à l'aide d'une règle plus ou moins trouvée/inventée (Vayre, 2013b). Et, du point de vue du parcours informationnel de PierreAndré, c'est plutôt selon ce type de raisonnement que ce dernier retrouve progressivement l'antécédent (i.e. : la campagne marketing) du conséquent que constitue le pic YouTube.

Compte tenu que les interfaces des TBDM sont généralement conçues selon des principes ergonomiques relativement identiques, ces dernières tendent donc à favoriser des processus de construction des connaissances plus exploratoires qui consistent, en quelque sorte, à expérimenter différentes associations de données afin de remonter à une réalité qui n'est pas directement observable (Auray, 2011 ; Ginzburg, 1989). En d'autres termes, compte tenu que l'architecture des TBDM ont pour fonction de redistribuer les activités de traitement et d'analyse des données de façon à lever certaines inerties organisationnelles et que l'ergonomie de leurs interfaces instaure un rapport plus exploratoire à l'enquête, ces dernières 
tendent à favoriser des processus de construction de connaissance plus sérendipitaires (Merton et Barber, 2004).

En outre, les TBDM sont conçus de façon à encourager le traitement et l'analyse collective des données. Par conséquent, ces derniers peuvent également contribuer à instituer au sein de l'organisation un rapport à la connaissance qui, à la fois moins rigide, moins conventionnel et plus collaboratif, tend également à favoriser une certaine disposition à la perplexité ${ }^{7}$ (Stark, 2009).

\section{POUR UN NOUVEAU MANAGEMENT DE L'INNOVATION ?}

Sur le plan organisationnel, les TBDM recouvrent donc un projet d'innovation sociotechnique qui, une fois déployé au sein de l'entreprise, implique des changements cognitifs importants. Car, comme nous l'avons vu, les TBDM favorisent des processus de construction des connaissances qui sont à la fois plus flexibles et exploratoires aux niveaux individuel et collectif. Et, nous l'avons également déjà dit, une fois implémentés au sein de l'organisation, les TBDM composent une sorte de réseau de nœuds d'information relativement ouverts qui tendent à encourager la sérendipité des utilisateurs (Merton et Barber, 2004).

Cependant, en permettant l'exploitation de nouvelles logiques d'exploration des données (March, 1991 ; Mothe et Brion, 2008), les TBDM ne sont pas, en soi, très intéressants pour l'entreprise. L'avantage concurrentiel qui découle de leur implémentation est plus pragmatique. Comme nous l'avons plusieurs fois mis en avant, les TBDM permettent effectivement d'explorer plus finement les usages que les travailleurs et les consommateurs font des systèmes de production et des produits/services de consommation. Or, les activités d'exploration des usages sont, cette fois-ci, de réelles sources d'avantage concurrentiel compte tenu qu'elles constituent une part importante du processus d'innovation (Mallard, 2011).

\footnotetext{
${ }^{7}$ Selon David Stark (2009), les situations de perplexité renvoient à des situations de remise en cause qui consistent moins à confronter des valeurs conventionnellement établies (Boltanski et Thévenot, 1991) qu'à rechercher à définir ce qu'est la valeur d'une chose. En référence aux travaux de Luc Boltanski et Laurent Thévenot (1991), la perplexité relève donc moins d'une mise en confrontation des cités domestiques, marchandes, industrielles, etc., que d'une remise en cause des principes d'équivalence qui y sont associés. C'est pourquoi, pour David Stark (2009), il existerait une sorte de cité de la perplexité où le grand est celui qui sait mettre à l'épreuve la notion même de grandeur; c'est-à-dire, qui sait créer et/ou traiter des situations de réelle indétermination. Notons alors que, toujours selon cet auteur, la perplexité, qui est au cœur du principe de l'hétérarchie, tend à favoriser des modèles organisationnelles plus flexibles et réactifs aux fluctuations du marché.
} 
Par conséquent, dans l'objectif de mieux saisir les avantages et les inconvénients qui sont associés aux usages des TBDM, nous proposons dans la section 4.1 de dégager trois grands modèles d'exploration des usages : l'incubation, la percolation et l'internalisation (Mallard, 2011). Nous verrons que dans le domaine de l'innovation de produit/service, les TBDM tendent plutôt à favoriser les modèles de la percolation et de l'internalisation (section 4.2). Ensuite, nous montrons que dans le domaine de l'innovation organisationnelle, les TBDM tendent à avantager le modèle de la percolation (section 4.3). Finalement, nous soulignons que si les TBDM peuvent permettre aux entreprises d'améliorer leur processus d'innovation incrémentale, il est néanmoins possible de questionner leur apport dans le cadre de processus d'innovation plus radicale (section, 4.4).

\subsection{Explorer les usages : incubation, percolation et internalisation}

De façon très schématique, à travers le développement des technologies big data, les TBDM permettent aux organisations de tisser des relations plus intimes avec leurs environnements interne (i.e. : l'organisation) et externe (i.e. : le marché).

Comme nous l'avons annoncé en introduction, les TBDM ont pour principales fonctions de représenter les traces d'usage que les travailleurs et les consommateurs fournissent durant leurs activités de production et de consommation. Autrement dit, les TBDM doivent faciliter l'exploration de ces traces d'usage de façon à améliorer les processus d'innovation que l'entreprise déploie dans le domaine des biens et des services mais aussi dans celui de l'organisation.

Afin de bien comprendre comment les TBDM peuvent favoriser l'atteinte cet objectif, nous souhaitons revenir sur les trois dynamiques d'exploration des usages que sont l'incubation, la percolation et l'internalisation (Mallard, 2011) :

- Dans le cas de l'incubation, l'exploration des usages est réalisée à l'intérieur de l'entreprise. Ici, différents spécialistes (i.e. : sociologue, ergonomes, économistes, etc.) s'associent dans le but de tester un produit/service sur une population restreinte afin d'expérimenter un ensemble d'hypothèses et ainsi donner forme à des usages qui auront pour finalité d'orienter la conception. Ensuite, le produit/service est lâché dans la nature à travers sa commercialisation (Mallard, 2011).

- Dans le cas de la percolation, les univers de la conception et de l'utilisation sont plus perméables que dans celui de l'incubation. Il s'agit ici de rester à l'écoute des consommateurs de façon à pouvoir saisir leurs usages sans pour autant les avoir 
organisés par le biais d'épreuves préconfigurées (Mallard, 2011). La percolation permet ainsi d'explorer les usages durant les phases de conception et de commercialisation du produit/service.

- Dans le cas de l'internalisation, les objectifs sont cette fois-ci de capter les usages déployés par les communautés de pratique de façon à récupérer les inventions développées par certains de leurs utilisateurs (Von Hippel, 2005) dans l'objectif de les retravailler puis de les rediffuser, à une échelle différente, à l'intérieur du marché (Mallard, 2011).

\subsection{Les TBDM et l'innovation de produit/service par percolation et internalisation}

Dans le domaine de l'innovation de produit/service, il ressort alors que les TBDM favorisent les deux modèles d'exploration des usages que sont la percolation et l'internalisation.

Nous l'avons déjà plusieurs fois mentionné, avec le développement toujours croissant de l'électronisation du commerce (Rallet, 2001), les consommateurs sont conduits à mobiliser les TIC durant leurs activités de consommation. Par voie de conséquence, le mouvement actuel de numérisation du commerce favorise l'exploration des usages par percolation puisque les consommateurs, en faisant un usage toujours plus important des TIC, produisent de grandes quantités d'informations qui sont riches d'enseignement pour les concepteurs (Mallard, 2011). En d'autres termes, les consommateurs deviennent ici des sortes d'explorateurs dont les usages font l'objet d'analyses continuent de manière à permettre aux offreurs d'améliorer leurs processus d'innovation de produit/service. C'est précisément selon ce type de rétroaction que, comme l'illustre le verbatim ci-dessous, un prestataire peut proposer à un client bien identifié de nouveaux services susceptibles de l'intéresser (Boullier, 1997).

«Aujourd'hui, quand un client appelle on peut, à partir de son numéro de téléphone avoir une fiche client qui dit que celui-ci adore les suites, qu'il a un panier moyen de 800 euros par nuitée, qu'il a posté une réclamation récemment dans un établissement parce qu'il avait une petite remarque sur un sujet, et qu'on lui a apporté des réponses dont il était très content. Donc, ça nous permet aujourd'hui, quand un client appelle, de mieux le connaitre et de lui proposer tout de suite un produit qui doit mieux lui correspondre. » (Retour d'expérience, Peter, Directeur Commercial Opérations \& e-business, réseaux d'hôtels et de restaurants Hôtes de Voyage, 2014) 
Comme nous l'avons pointé dans la section 1 ( $c f$. le cas de Carblue) et dans la section 2 ( $c f$. le cas de ViewAnalytics), les TBDM ont pour fonction de représenter les usages que les consommateurs font des biens et/ou des services proposés par l'entreprise. En ce sens, et en référence à la section 2, ils permettent aux managers d'exploiter de nouvelles logiques d'exploration des usages que les consommateurs font des innovations de produit/service de façon à orienter et/ou réorienter, à tout moment, une nouvelle/une ancienne conception.

En outre, à travers la puissance de captation, de stockage et de traitement que recouvrent les technologies big data, les TBDM permettent également de représenter les usages qui sont développés au sein des communautés de pratique (Von Hippel, 2005). Par le biais de différentes sources textuelles, photographiques, audio ou encore vidéo, ils peuvent permettre de repérer certaines tendances d'innovation issues d'un groupe de praticiens considérés comme innovants.

«Je vais prendre un exemple, dans le domaine de la mode [...]. Il y a une start-up, à New-York, qui, à partir des images vidéo des défilés de mode, analyse donc les données non-structurées, analyse la forme des vêtements, la couleur, le style, pour en tirer automatiquement des données structurées qui vont servir aux marques de prêt-à-porter pour pouvoir construire un projet de collection. »(Présentation, Gislain, Fondateur du magazine spécialisé dans l'informatique décisionnel DecIT, 2014)

Bien entendu, le cas exposé ci-dessus est bien particulier. En ce sens, il ne constitue pas une application commercialisée des TBDM qui permettent le plus souvent d'examiner les fils de discussions réalisés sur les réseaux sociaux, les sites spécialisés, les blogs, etc. Néanmoins, ce cas est intéressant car il rend compte des potentialités techniques des TBDM et de la diversité des applications qui peuvent en découler. À tout le moins, ce cas de figure illustre bien comment les applications des TBDM les plus innovantes peuvent constituer d'intéressants outils d'exploration des usages par internalisation.

\subsection{Les TBDM et l'innovation organisationnelle par percolation}

Dans le domaine de l'innovation organisationnelle, les TBDM favorisent les formes d'exploration des usages par percolation. Car, comme nous l'avons également plusieurs fois précisé, si les TBDM permettent de rendre visibles et intelligibles les traces d'usage issues du marché, elles permettent également de représenter celles issues de l'organisation. 
Par exemple, dans le cas du secteur de la logistique, en améliorant la visibilité des processus de stockage et de livraison des marchandises, les TBDM peuvent permettre d'instaurer de nouvelles formes de management de la chaîne logistique qui sont qualifiées de «gestion par exception ».

«Donc, il est très important pour le supply chain manager de pouvoir suivre de manière précise ce qui se passe [...] dans la chaîne de valeur. Avant, dans la logistique, ils avaient un certain nombre d'alertes qui étaient identifiées par le système [...]. Mais, le problème était que ces alertes n'étaient pas suffisamment qualifiées pour permettre aux opérationnels de réagir. Grâce à l'implémentation des technologies in-memory analytics, prenons l'exemple d'une grève sur le port du Havre, on va immédiatement identifier quels sont les flux logistiques critiques qui vont impacter le niveau d'engagement et qui vont amener des pénalités. On va donc être capable d'apporter à l'opérationnel l'outil qui va lui permettre de faire des simulations pour identifier le flux logistique qu'il va falloir réacheminer d'une manière différente [...]. Il va pouvoir connaitre l'impact sur ces objectifs opérationnels et ainsi pouvoir agir de manière proactive en choisissant le scénario qui est le plus optimisé. [...] L'intérêt est donc ici de faire de la gestion par exception, de réagir à partir d'un certain nombre d'alertes qui sont remontées [...]. » (Présentation, Odelia, Sales Excutive, éditeur de logiciels décisionnels WT Quintet, 2014)

Le cas de la maintenance constitue également une bonne illustration des innovations organisationnelles que peuvent apporter les TBDM. Afin d'illustrer cet aspect, Gabriel, PDG de la société de conseils et de services Genicap, propose d'examiner le cas fictif du réacteur d'avion. Par le biais d'un système de captation adapté, les TBDM pourraient selon lui permettre de rendre visibles et intelligibles les processus d'usure du réacteur de façon à simuler, prédire et anticiper les opérations de maintenance qui doivent y être associées.

\subsection{Les TBDM favorisent-ils l'innovation incrémentale ou l'innovation radicale?}

Ces différents exemples montrent bien comment, en tant qu'instruments de management de l'innovation, les TBDM peuvent avantager les processus d'innovation incrémentale (Freeman, 1994). Car, en favorisant les formes d'exploration des usages dites externes ( $c f$. le modèle de la percolation et de l'internalisation), les TBDM permettent aux concepteurs de mieux saisir les pratiques, les attentes et les besoins des travailleurs et/ou des consommateurs. De cette façon, ils permettent de conférer aux utilisateurs une réelle autorité cognitive (Baiocchi et 
Forest, 2014 ; Wilson, 1983). En d'autres termes, en levant un certain nombre de frontières séparant traditionnellement l'univers de la conception et celui de l'utilisation, les TBDM instaurent de nouvelles relations entre innovation et usage : les deux sont plus intimement associés. C'est précisément en ce sens que les TBDM favorisent les processus d'innovation incrémentale puisqu'ils facilitent l'exploration des comportements des utilisateurs afin de permettre aux concepteurs de réaliser rapidement des évolutions technologiques ( $c f$. Greiner, 1998) dans le but d'adapter plus étroitement l'innovation aux usages réels (Akrich et al., 2006).

Néanmoins, il est possible de questionner l'apport des TBDM dans le cadre d'une stratégie d'innovation radicale (Freeman, 1994). En effet, il ne faut pas oublier qu'une innovation de rupture suppose une certaine distanciation de la part des concepteurs (Mallard, 2011). Car, dans ce cas de figure, les innovateurs cherchent moins à répondre au problème de l'adaptation de la conception aux usages qu'à celui de la création des usages à travers la commercialisation d'une conception radicalement nouvelle. Aussi, en tant qu'outils de gestion du processus d'innovation, les TBDM n'entravent pas, bien entendu, les stratégies de rupture. En effet, il peut être intéressant de bien connaitre les usages afin de chercher à se positionner en décalage. Ou encore, une fois l'innovation de rupture mise sur le marché, les TBDM peuvent permettre de mieux comprendre les usages qui en sont fait et donc de mieux l'adapter à ces derniers. Nous retrouvons alors le processus d'innovation incrémentale. C'est pourquoi, dans le cas d'une innovation de rupture, les TBDM ne sont pas inutiles. Néanmoins, elles ne sont pas suffisantes : «créer les usages » n'est pas la même chose qu' «adapter aux usages ». Dans le premier cas, il ne suffit pas de bien comprendre les pratiques, les attentes et les besoins des utilisateurs mais plutôt de les inventer. Et, c'est précisément en ce sens que, en tant qu'instruments de management de l'innovation, il est possible de questionner l'apport des TBDM pour ceux qui souhaitent innover d'une façon réellement radicale.

\section{CONCLUSION}

Les TBDM sont une innovation de produit sur le marché des technologies big data. Cependant, du point de vue de l'entreprise qui les implémente, ils sont également une innovation sociotechnique qui implique des changements culturels et organisationnels importants. Une fois adoptés par l'organisation, les TBDM donnent forme à une architecture informationnelle qui implique la mise en place de nouveaux attachements entre les différentes unités opérationnelles et décisionnelles de l'entreprise (section 2). 
Plus précisément, cette architecture constitue un réseau composé de nœuds d'information qui, par le biais des interfaces que sont les TBDM, permettent l'institution de nouveaux attachements entre l'entreprise (i.e. : l'organisation) et son environnement (i.e. : le marché). Et, en multipliant et facilitant les possibilités de croisement entre les données internes et externes à l'entreprise, ces nœuds constituent autant d'espaces informationnels qui favorisent une certaine disposition à la sérendipité et à la perplexité (section 3 ; Merton et Barber, 2004 ; Stark, 2009).

De ce fait, les TBDM permettent finalement aux managers de l'entreprise d'exploiter de nouvelles stratégies d'exploration des usages que les consommateurs ou les travailleurs font des produits/services de consommation et des systèmes de production (March, 1991 ; Mothe et Brion, 2008). C'est d'ailleurs ici que se trouve tout leur intérêt : en soutenant les activités d'exploration des usages, les TBDM doivent permettre à l'entreprise d'améliorer ses processus d'innovation (Mallard, 2011). Il ressort alors que dans le domaine de l'innovation de produit/service, les TBDM encouragent plutôt les modèles d'exploration des usages qui sont de l'ordre de la percolation et de l'internalisation. Et, dans le domaine de l'innovation organisationnelle, les TBDM avantagent plutôt le modèle de la percolation (section 4).

En conclusion, les TBDM sont des outils de management de l'innovation intéressants. Comme nous l'avons vu, ils peuvent effectivement aider les entreprises à améliorer la performance de leurs processus d'innovation incrémentale. Cependant, comme nous l'avons souligné dans la section 4.4, il est possible de questionner leur apport dans le cadre de processus d'innovation de rupture. Aussi, si nos travaux ont permis de faire émerger ce questionnement, ils n'offrent que très peu d'éléments permettant de le traiter concrètement. Nos investigations doivent donc être prolongées afin de mieux saisir la manière dont les TBDM peuvent avantager ou non les processus d'innovation radicale. 


\section{Références}

AKRICH, M., CALlON, M. et LATOUR, B. 2006, Sociologie de la traduction : Textes fondateurs, Paris, Presses des Mines.

ALLEN, T. J. and HENN, G. W. 2007, The Organization and Architecture of Innovation. Managing the Flow of Technology, Burlington, Butterworth-Heinemann.

AURAY, N. 2011, «Les technologies de l'information et le régime exploratoire », in: ANDEL, (van) P. et BOURCIER, D. (dir), La sérendipité. Le hasard heureux, Paris, Herman, p. 329-343.

BAIOCCHI, M. C. et FOREST, D. 2014, «L'usager comme autorité cognitive. Perspectives théoriques sur les systèmes de recommandation », Les Cahiers du Numérique, vol. 10, $\mathrm{n}^{\circ} 1, \mathrm{p}$. 127-157.

BARREY, S. 2007, «Struggling to be displayed at the point of purchase: the emergence of merchandising in French supermarkets », in : CALLON, M., MILLO, Y. and MUNIESA, F. (dir.), Market Devices, Malden, Blackwell Publishing, p. 92-108.

BÉGUIN, P. and RABARDEL, P. 2000, «Designing for instrument-mediated activity », Scandinavian Journal of Information Systems, $\mathrm{n}^{\circ} 12$, p. 173-190.

BERNARD, S. 2012, Travail et automatisation des services. La fin des caissières?, Toulouse, Octarès.

BOLTANSKI L. et THÉVENOT L., E. 1991, De la justification : les économies de la grandeur, Paris, Gallimard.

BOULLIER, D. 1997, «Les usages comme ajustements : services propriétaires, moteurs de recherche et agents intelligents sur Internet », Penser les usages, Bordeaux-Arcachon.

BOUSSARD, V. 2001, «Quand les règles s'incarnent. L'exemple des indicateurs prégnants », Sociologie du travail, vol. 43, n 4, p. 533-551.

BOUTANG, Y. M. 2007, Le capitalisme cognitif. La Nouvelle Grande Transformation, Paris, Amsterdam.

CHIAPELlO, E. et GILBERT, P. 2013, Sociologie des outils de gestion. Introduction à l'analyse sociale de l'instrumentation de gestion, Paris, La Découverte. 
COCHOY, F. (dir.) 2004, La captation des publics : "c'est pour mieux te séduire mon client... », Toulouse, Presses Universitaires du Mirail.

CONINK, (de) F. 2004, «Vers la construction d'un nouveau système ? Du post-taylorisme à l'effritement des organisations », Travail et Emploi, n ${ }^{\circ}$ 100, p. 139-149.

DEJOURS, C. 2003, L'Évaluation du travail à l'épreuve du réel. Critique des fondements de l'évaluation, Paris, INRA Éditions.

DESROSIÈRES, A. et THÉVENOT, L. 1988, Les Catégories socioprofessionnelles, Paris, La Découverte.

DIMAGGIO, P. and POWELL, W. 1983, «The iron-cage revisited: institutional isomorphism and collective rationality in organizational field », American Sociological Review, $\mathrm{n}^{\circ}$ 48, $\mathrm{p}$. 147-160.

FRAENKEL, B. 1995, «La traçabilité. Une fonction caractéristique des écrits de travail », Connexions, $\mathrm{n}^{\circ} 65$, p. 63-75.

FREEMAN, C. 1994, «The economics of technical change », Cambridge Journal of Economics, vol. 18, $\mathrm{n}^{\circ}$ 5, p. 436-514.

GINZBURG, C. 1989, Mythes, emblèmes, traces. Morphologie et histoire, Verdier, Lagrasse.

GLASER, B. and STRAUSS, A. 1967, The Discovery of Grounded Theory. New-York, Aldine Publishing Company.

GREINER, L. E. 1998, «Evolution and Revolution as Organizations Grow », Harvard Business Review, May-June.

HALPERN, C. et LE GALÈS, P. 2011, «Pas d'action publique sans instruments propres », Revue française de science politique, vol. $61, \mathrm{n}^{\circ} 1, \mathrm{p} .51-78$.

KESSOUS, E. 2012, L'attention au monde. Sociologie des données personnelles à l'ère numérique, Paris, Armand Colin.

LATOUR, B. 1993, «Le «pédofil » de Boa Vista - montage photo-philosophique », in :

LATOUR, B. (dir.), Petites leçons de sociologie des sciences, Paris, La Découverte, p. 171225.

LOISEL, J.-P. 2000, «Vers une sociologie de l'Intranet? », AXIALES L'Esprit projet, p. 1-3. 
MALLARD, A. 2011, «Explorer les usages : un enjeu renouvelé pour l'innovation des TIC », in : DENOUEL, J. et GRANJON, F. (Dir.), Communiquer à l'ère numérique. Regards croisés sur la sociologie des usages, Paris, Presses des Mines, p. 253-282.

MARCH, J. G. 1991, «Exploration and Exploitation in Organizational Learning », Organization Science, vol. 2, $\mathrm{n}^{\circ}$ 1, p. 71-87.

MERTON, R. K. and BARBER, E. G. 2004, The Travels and Adventures of Serendipity: A Study in Sociological Semantics and the Sociology of Science, Princeton, Princeton University Press.

MILLE, A. 2013, « De la trace à la connaissance à l'ère du Web. Introduction au dossier », Intellectica, vol. $1, \mathrm{n}^{\circ} 59$, p. 7-28.

MOTHE, C. et BRION, S. 2008, «Innovation : exploiter ou explorer? », Revue Française de Gestion, $\mathrm{n}^{\circ} 187$, p. 101-108.

NAVILLE, P. 1961, L'automation et le travail humain. Rapport d'enquête (France 19571959), Paris, Centre National de la Recherche Scientifique.

ORLIKOWSKI, W. 1992, «The duality of technology: rethinking the concept of technology in organizations », Organization Science, vol. 3, n 3, p. 398-427.

PEIRCE, C. S. 1974, Collected Papers, Cambridge, Harvard University Press.

RALLET, A. 2001, « Commerce électronique ou électronisation du commerce », Réseaux, vol. $2, \mathrm{n}^{\circ} 106$, p. 17-72.

REYNAUD, J.-D. 2001, « Le management par les compétences : un essai d'analyse », Sociologie du travail, vol. $43, \mathrm{n}^{\circ} 1, \mathrm{p} .7-31$.

RICCIO, P.-M. et BONNET, D. 2011, TIC et innovation organisationnelle. Journées d'études MTO 2011, Paris, Presses des Mines.

STARK, D. 2009, The Sense of Dissonance. Accounts of Worth in Economic Life, Princeton, Princeton University Press.

TERSSAC, (de) G. 2002, Le Travail : une aventure collective, Toulouse, Octarès.

VAYRE, J.-S. 2013a, «Le big data et la relation client. Quand les traces numériques organisent l'échange marchand», 12e Journées Normandes de Recherche sur la Consommation : Société et consommation, Caen. 
VAYRE, J.-S. 2013b, «Découvrir l'offre dans un contexte de distribution multi-canal. Exploration autour de la sérendipité », Journée d'étude de l'IDHE : "Marchés, organisations, institutions : regards croisés sur la sociologie et l'économie », Cachan.

VAYRE, J.-S. 2014, «Manipuler les données. Documenter le marché. Les implications organisationnelles du mouvement big data », Les Cahiers du Numérique, vol. 10, n 1, p. 95125.

VON HIPPEL, E. 2005, Democratizing innovation, Cambridge, MIT Press.

WILSON, P. 1983, Second-hand knowledge. An inquiry into cognitive authority. Westport, Greenwood Press. 